\title{
CME quiz
}

The purpose of this quiz is to provide a convenient means of self-assessment of your reading of the scientific content of this issue of JAOA. Enter your answers to the questions in the spaces provided so that you can easily check them with the answers that will be published next month.

To apply for CME credit, transfer your answers to the mail-in card on page 19 and return it to the CME office. So that you may complete this self-assessment in privacy, use only your member number to apply for CME credit. The CME office will record only the fact that you have completed the self-assessment test. Any grading will be done by the Editorial Department only for the purpose of planning areas of study which may be helpful to cover in future issues of JAOA.
1. The estimated incidence of horseshoe kidney is which of the following?
(a) 1 in 200
(b) 1 in 400
(c) 1 in 800
(d) 1 in 1,000

2. Routine aortography remains the procedure of choice for diagnosis of horseshoe kidney.
(a) True
(b) False

3. Papillotomy (sphincterotomy) of the ampulla of Vater has been shown to be effective treatment for pancreas divisum because it allows adequate drainage through the duct of Wirsung.

(a) True

4. Pancreas divisum results from the failure of fusion of the dorsal and ventral elements of the pancreas during the second month of gestation.

$$
\text { (a) True }
$$$$
\text { (b) False }
$$

5. Which of the following statements regarding capillary hemangiomas is true?

(a) It needs early treatment in all cases because it will not regress by itself. (b) It shows contrast enhancement on computed tomography.

(c) It sometimes is associated with proptosis.

(d) It occurs more often in men than women.

6. The clinical differential diagnosis of capillary hemangiomas encompasses all orbital condi- tions that can affect children. They include chiefly which of the following?

(a) Inflammation

(b) Hemorrhage

(c) Lymphangioma

(d) Neurofibromatosis

(e) Rhabdomyosarcoma

(f) All of the above 